\title{
Glycyrrhizic Acid as a Novel Drug Delivery Vector: Synergy of Drug Transport and Efficacy
}

\author{
N.E. Polyakov* and T.V. Leshina
}

Institute of Chemical Kinetics and Combustion, Institutskaya Str., 3, Novosibirsk, 630090, Russia

\begin{abstract}
Supramolecular drug delivery systems (DDS) are widely used in pharmacology during last decades for designing new preparations with improved properties. Most of fundamental and applied research in this field has been devoted to the inclusion complexes with cyclodextrins (CD), which are assumed to possess protective properties and to decrease the hydrophobic behavior of the included molecules. At present a new generation of DDS has been developing. Novel DDS are focused simultaneously on several purposes, including improved bioavailability and stability of drugs, targeted drug delivery, and enhanced activity. One of the compounds that appear to be promising is glycyrrhizic acid (or glycyrrhizin). A number of experimental facts of high therapeutic activity of glycyrrhizin conjugates have been published, but no understanding "how it works" was presented. This manuscript will highlight our attempts to answer this question using physicochemical approach, and also outline the challenges and future opportunities for medical applications of glycyrrhizin conjugates.
\end{abstract}

Keywords: Glycyrrhizic acid, drug conjugates, drug delivery, novel delivery systems.

\section{INTRODUCTION}

It is known, that many drugs with useful activity profiles can exhibit very poor biodistribution properties, high toxicity, or low stability. One method that has been developed to assist drug formulations is the synthesis of inclusion complexes of drug molecules. By attaching drugs to a suitable carrier, the bioavailability of the drug can be maximized due to the increased solubility and circulation time. The stability of the drugs can be improved by the protection of "guest" molecule from the interaction with other active molecules, free radicals or metal ions. The absence of side reactions will result also in reduction of drug toxicity. The therapeutic efficacy of the drugs can be improved because of the facilitated transportation of the drugs to the desired tissues or organs. Several other mechanisms of influence of complexation on the reactivity of the guest molecule exist and will be also discussed in the present review.

Most of examples of drug delivery systems (DDS) have been devoted to the inclusion complexes with cyclic oligosaccharides - cyclodextrins (CDs), which are assumed to decrease the hydrophobic behavior of the included molecules [1-5]. Cyclodextrins have lipophilic inner cavities and hydrophilic outer surfaces, and are capable to interact with a large variety of guest molecules to form noncovalent inclusion complexes. They contain 6-8 D-(+) glucopyranose units attached by $\alpha-(1,4)$ glucosidic bonds. These natural CDs, $\alpha-\beta-$-, and $\gamma$-CDs (with 6,7 , or 8 glucose units respectively), differ in their ring size and solubility. $\beta-C D$ has been widely used in the early stages of pharmaceutical applications of inclusion complexes because of its ready

*Address correspondence to this author at the Institute of Chemical Kinetics and Combustion, Institutskaya Str., 3, Novosibirsk, 630090, Russia; Tel: 7(383) 333-2947; Fax: 7(383) 330-7350; E-mail: polyakov@kinetics.nsc.ru availability and cavity size suitable for the widest range of drugs. But the low aqueous solubility and nephrotoxicity limited the use of $\beta-\mathrm{CD}$ especially in parenteral drug delivery [6].

A new generation of DDS possesses simultaneously of several useful properties. In addition to improved bioavailability, new drug conjugates provide targeted drug delivery to the desired tissues or organs, and enhance drug activity. $\beta$-Glycyrrhizic acid (GA), a triterpene glycoside from Glycyrrhiza uralensis Fisher (Fabaceae), appears to be promising drug carrier of new generation [7-8]. It was recently demonstrated that glycyrrhizic acid reduces toxicity and increases therapeutic activity of molecular complexes formed by several important anti-inflammatory [9-13] and cardiovascular preparations [14-16]. For example, the complexes of GA with nifedipine and lappaconitine, blockers of ion channels, shows significant increase in their therapeutic activity on the models of adrenaline-induced hypertension and $\mathrm{CaCl}_{2}$-induced arrhythmia. Availability of excellent review of pharmaceutical activity of GA conjugates spares us the necessity to discuss these examples here [17]. The main question which is shelved in these papers is the mechanism of influence of complexation on therapeutic activity of drugs. From our viewpoint, to understand the reasons underlying the abovementioned interplay between toxicity and therapeutic activity, one should explore the molecular-level mechanisms of the impact of complexation on drug activity. In this review we will summarize our attempts to clarify this problem using various physicochemical methods.

\section{SELF-ASSOCIATES OF GLYCYRRHIZIC ACID AS A PRECURSOR OF DRUGS CONGUGATES}

$\beta$-glycyrrhizic acid (GA) is an amphoteric compound which contains both hydrophilic (glucuronic acid) and hydrophobic (glycyrrhetic acid) fragments (Fig. 1). 


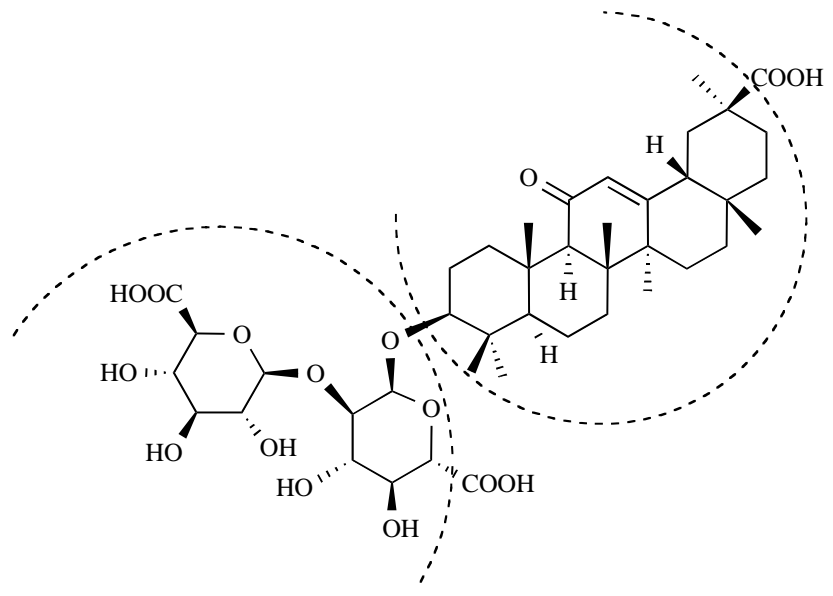

Fig. (1). Structure of glycyrrhizic acid.

Due to amphoteric character of GA molecule, it manifests the properties typical for micelle-forming substances [18-23]. According to these studies, the critical micellar concentration (CMC) of GA in water solution is 1 $\mathrm{mM}$. Also, several examples appeared which demonstrate the increase of water solubility of some low soluble drugs in GA solution above CMC [24-26]. However, these works did not discuss the ability of glycyrrhizic acid to form complexes with other molecules. For the first time GA complex formation was widely discussed in [27] from the influence of GA on the therapeutic activity of some drugs at concentration below CMC, however, no evidences of complex formation were presented. Ten years later Romanenko and Murinov [23] had measured the viscosity behavior of GA solution, and found some interesting features. The most important finding was the presence of two critical points on concentration dependence at $2 \times 10^{-5} \mathrm{M}$ and $1 \times 10^{-3} \mathrm{M}$ where the viscosity changed in discrete steps. The second point corresponds to $\mathrm{CMC}$, but nothing was known at that moment about the nature and structure of GA associates at low concentrations. Some additional information on the behavior of glycyrrhizin solution was obtained from nuclear magnetic resonance (NMR) experiments, including relaxation measurement [28-29]. NMR techniques are widely used in the investigation of cyclodextrins inclusion complexes due to high sensitivity to the molecular dynamics and environment [30]. It was observed that increasing GA concentration causes a substantial line broadening which is unambiguously related to the acceleration of spin-spin relaxation $\left(\mathrm{T}_{2}\right)$ of the GA protons. It is known that in liquids the rate of nuclear $T_{2}$ relaxation depends on the rotation correlation time of the molecule to which the nuclei under study belong [31-33]. The rotation correlation time depends, in turn, on the molecule size which can vary substantially upon self-association or complex formation. It was revealed that the time-dependence of the integral intensity of echo signal is well described only by biexponential function: $\mathrm{A}(\mathrm{t})$ $=\mathrm{P}_{1} \cdot \exp \left(-\mathrm{t} / \mathrm{T}_{21}\right)+\mathrm{P}_{2} \cdot \exp \left(-\mathrm{t} / \mathrm{T}_{22}\right)$. Usually observation of biexponential relaxation in micellar solutions is assigned to the difference in the relaxation rates of the protons belonging to the same type and located in an associate and in solution in the non-associate state. In this case the relaxation times $\mathrm{T}_{21}$ and $\mathrm{T}_{22}$ refer to the molecules in solution and the associate, and $\mathrm{P}_{1}$ and $\mathrm{P}_{2}$ are the probabilities to find a molecule in the non-associated (mainly monomer) and associate states, respectively [33]. The behavior of the parameters $\mathrm{P}_{1}$ and $\mathrm{P}_{2}$ for methyl protons of glycyrrhizin is typical for micellar solutions: $\mathrm{P}_{2}$ grows linearly with GA concentration, and $\mathrm{P}_{1}$ is constant in the concentration range 0.5-2 mM [28]. NMR study confirms the previous conclusion on the glycyrrhizin micelles formation at the concentration range above $1 \mathrm{mM}$. The characteristic relaxation times $T_{21}$ and $T_{22}$ were calculated and equal 50 and $10 \mathrm{msec}$ correspondingly. Short relaxation time of glycyrrhizin protons in micellar solution results in broadening NMR lines in the spectra. The same visual effect was observed under incorporation of "guest" molecule into GA micelle [28-29]. The intrigue is the relaxation time of "guest" protons is less than for free molecule even at low GA concentration $(<1 \mathrm{mM})$ [34].

More detailed information on GA self-associates was obtained from the measurements of relaxation time of GA protons in the aqueous solution with different $\mathrm{pH}$ and in non aqueous solutions. No micelles were detected in pure methanol solution or methanol-water mixture (> $40 \%$ of water) [29]. Also, micelle formation was detected only in acid and neutral aqueous solutions, no changes in relaxation time were observed at $\mathrm{pH}>8$. Interestingly, the $\mathrm{T}_{2}$ relaxation time of methyl protons is near $140 \mathrm{msec}$ in all concentration range $0-2 \mathrm{mM}$. This value is higher than previously mentioned $50 \mathrm{msec}$ for $\mathrm{T}_{21}$. We propose that $\mathrm{T}_{2}=140 \mathrm{msec}$ belongs to monomers of GA, but $\mathrm{T}_{21}=50 \mathrm{msec}$ belongs to "pre-micellar" state. An important conclusion which can be drawn from these studies is that the driving force of premicellar associates formation is hydrogen bonding between acid groups of two (or more) GA molecules. These associates can form inclusion complexes with various drugs and are stable at low and neutral $\mathrm{pH}$. In the next section of this review we will show that in contrast to GA micelles (as well as CD complexes), GA conjugates with different drugs exist even in non aqueous solutions, and the stoichiometry of these conjugates will be determined.

\section{CAROTENOID CONJUGATES OF GLYCYR- RHIZIC ACID}

Carotenoids are known as a class of natural pigments and antioxidants (see Fig. 2 as an example of carotenoids structure). Recently much attention has been focused on the reactions between carotenoids and free radicals [35-37] because of the ability of carotenoids to prevent the development of various diseases caused by toxic free radicals, including infarction, cerebral thrombosis, and tumors [38-39]. Carotenoids are assumed to protect cells by scavenging either free radicals or excited oxygen that have a severe impact on cells. At the same time, wide practical application of carotenoids as antioxidants is substantially hampered by their hydrophobic properties, instability in the presence of oxygen and high photosensitivity. One of the promising ways to overcome these problems is the preparation of supramolecular inclusion complexes. Application of inclusion complexes was first related to an attempt to minimize the aforementioned disadvantages of carotenoids when used in the food industry, cosmetology, and medicine [40]. Several attempts were made to prepare inclusion complexes of carotenoids with cyclodextrins, which are assumed to possess protective properties and to decrease the hydrophobic behavior of the included molecules 
[41-45]. Main imperfection of carotenoid-cyclodextrin complexes is their poor solubility. In reality, these complexes form water dispersions, rather than solutions. According to the studies of Mele with coauthors [41], carotenoid-cyclodextrin complexes in water form large aggregates with size 100-200 $\mathrm{nm}$. Recently we have presented several examples of water soluble complexes of $\beta$ carotene and some other carotenoids with glycyrrhizin [4647] and natural polysaccharide arabinogalactan [48-49], a branched polymer with molecular mass 15000-20000. Both these complexes show good water solubility and provide some additional advantages fruitful for practical application. In this review we will focus only on glycyrrhizin complexes.

\subsection{Stoichiometry and Stability of Carotenoid- Glycyrrhizin Complexes}

To calculate the stability constant and stoichiometry of GA complexes, the standard approach based on the dependence of the absorption and fluorescence spectra of the carotenoids on GA concentration was applied [46]. Note that in the case of carotenoid complexes using of optical methods is more convenient compared to NMR techniques due to low solubility of carotenoids even in water-alcohol solutions. The high extinction coefficients of carotenoids $\left(\sim 10^{5} \mathrm{M}^{-1} \mathrm{~cm}^{-1}\right)$ allow one to work with very low concentrations. The stoichiometry of the complexes was calculated using Job's plot of the dependence of the optical density of carotenoid solution on the mole fraction of carotenoid at fixed total concentration $([\mathrm{Car}]+[\mathrm{GA}]=\mathrm{const})$. The position of a maximum of the curve at $\mathrm{R}=[\mathrm{Car}] /([\mathrm{Car}]+[\mathrm{GA}])=0.33$ corresponds to the 1:2 ratio between carotenoid and GA molecules in the complex. To estimate the complex stability constant, the change in optical density of the solution was measured at a fixed concentration of carotenoids with varying GA concentration. Under certain experimental conditions ([Car] $<[\mathrm{GA}]$ ) the Benesi-Hildebrand plot can be used to estimate both the complex stability constant and the order of complexation from a single experiment:

$$
\mathrm{A} / \Delta \mathrm{A}-1=1 /[\mathrm{GA}]^{\mathrm{n}} \times 1 / \mathrm{K}
$$

Here $\Delta \mathrm{A}=\Delta \varepsilon \times[\mathrm{Car}]$, and $\mathrm{K}$ is the stability constant of the complex for reaction:

$$
\begin{aligned}
& \mathrm{Car}+\mathrm{nGA} \stackrel{\mathrm{K}}{\rightleftharpoons} \mathrm{CarGa}_{\mathrm{n}} \\
& \mathrm{K}=\left[\mathrm{CarGA}_{\mathrm{n}}\right] \\
& {[\mathrm{Car}] \times[\mathrm{GA}]^{\mathrm{n}}}
\end{aligned}
$$

The plot of $\mathrm{A} / \Delta \mathrm{A}$ versus $1 /[\mathrm{GA}]$ provides the linear dependence for a second-order reaction $(n=1)$. For a thirdorder reaction $(n=2)$, the linear dependence would be obtained in the coordinates of $\mathrm{A} / \Delta \mathrm{A}$ versus $1 /[\mathrm{GA}]^{2}$. In all experiments with carotenoid-GA complexes, the linear dependence was obtained for $n=1$ only. Taking into account the result of the analysis of Job's plot, it was concluded that the complex is formed in second order reaction between one carotenoid molecule and one dimer of glycyrrhizic acid (Fig. 2).

$$
\mathrm{Car}+\mathrm{GA}_{2} \stackrel{\mathrm{K}}{\rightleftharpoons} \mathrm{CarGa}_{2}
$$

Note that all this experiments were carried out at the concentration range 0.01-0.1 mM, much less CMC [46]. This information is extremely important for understanding the structure of "pre-micellar" self-associates of GA. The estimated value of stability constant $\mathrm{K}$ is equal $10^{4}\left( \pm 10^{3}\right)$ $\mathrm{M}^{-1}$ for the $\beta$-carotene- $\mathrm{GA}_{2}$ complex. The thermodynamics study shows that hydrophobic interaction is an important factor in the binding, and the complex formation follows considerable desolvaion of ligand molecules. There are several examples of thermodynamics study of molecular complexes formation of glycyrrhizic acid with various organic molecules [50-51]. These studies demonstrate that high stability constants of GA complexes can be attributed to the mutual effect of both enthalpy and entropy contribution to the complex formation.

The important advantage of GA complexes over CD complexes is their stability in non-aqueous solutions. There are several examples of the detection of GA complexes in dimethyl sulfoxide (DMSO), methanol and acetonitrile [34, 46, and 47]. In [46] canthaxanthin-GA complex was detected and characterized from the fluorescence changes in the presence of GA. The fluorescence method is widely used to study inclusion complexes of the "guest-host" type. There are many examples of an increase in fluorescence intensity for cyclodextrin complexes [52-54]. The luminescence of molecules imbedded in the supramolecular cavity is strengthened by the protection against quenching and other processes occurring in solution. The stability constant of canthaxanthin-GA complex in DMSO estimated in [46] has an order $1 \times 10^{4} \mathrm{M}^{-1}$. The surprising result of this study is the existence of critical point around $1 \mathrm{mM}$ of GA concentration where the stability constant was changed, although no micelles formation was detected in non-aqueous solutions (Fig. 2).

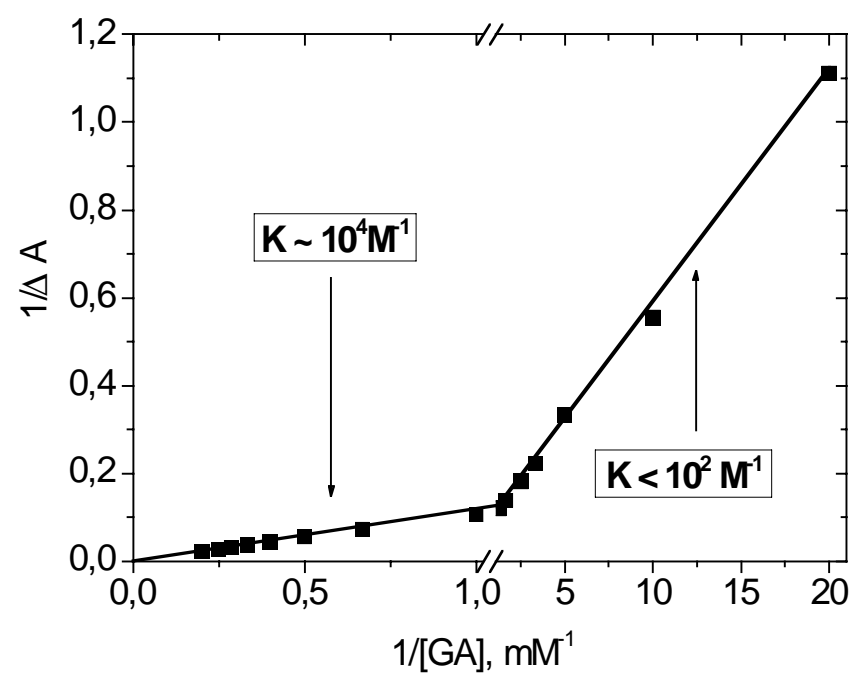

Fig. (2). Dependence of fluorescence intensity of carotenoid canthaxanthin (III) on GA concentration in DMSO solution containing $5 \%$ of water (adopted from [46]).

It might be suggested that at high concentration glycyrrhizin self-associates changes their structure from ring-like to more complex structures, and such restructuring results in less stable complexes. This effect might be the reason of several surprising observations of decreasing the therapeutic effect of GA complexes with some drugs with increasing GA concentration [55-56]. 


\subsection{Activity of Carotenoid-Glycyrrhizin Complexes}

In addition to structural information, an important aspect of the physicochemical approach is a study of drug reactivity in the complex. Such information will be helpful for deeper understanding the molecular mechanism of drug activity in the complex, its toxicity and stability.

We propose that the most important finding during investigation of carotenoids-GA complexes is the influence of complexation on the oxidation potentials of carotenoids [47]. This result has allowed us to explain the observations of significant effect of complexation on the rates of redox reactions of carotenoids [46-47], and provide a new insight into the mechanisms of therapeutic activity of GA complexes with other drugs. The measurement of the oxidation potential of two carotenoids zeaxanthin and canthaxanthin in the presence of GA shows the increase in $\mathrm{E}_{1 / 2}$ : by $0.05 \mathrm{~V}$ for canthaxanthin and by $0.03 \mathrm{~V}$ for zeaxanthin. Can these small changes affect the reactivity of carotenoids significantly? The answer is "yes". The important role of electron transfer was established for reactions of carotenoids towards metal ions, quinones and free radicals, which are closely related to their antioxidant activity as well as stability in living systems, food and medical preparations [46, 47, 57, 58]. As it was demonstrated in [58], the reaction with $\mathrm{Fe}$ ions can be considered as one of the possible mechanisms of the prooxidant activity of $\beta$-carotene. The significant decrease in reaction rate (more than one order in magnitude) was detected in the reaction of $\beta$-carotene with $\mathrm{Fe}^{2+}$ ions and quinones [46] in GA complexes. On the other hand, it was demonstrated using EPR (electron paramagnetic resonance) spin trapping technique, that the ability of carotenoids to scavenge hydroperoxyl radicals (antioxidant activity) increase with growth of their oxidation potential [57]. And indeed, an increase the oxidation potential of some carotenoids by the complexation with GA resulted in increase of their scavenging rate more than one order (Table 1) [47].

The $k_{C a r} k_{S T}$ values given in Table 1 were obtained by subtracting the GA contribution from the total rate constant measured in the presence of glycyrrhizic acid. From these values, it is clear that a synergetic effect of GA on the scavenging ability of some carotenoids occurs. Of importance is the absence of this effect for zeaxanthin. In [47] the authors have discussed this question in details. Analyzing the oxidation potentials of these three carotenoids and the dependence of the carotenoids scavenging rate on their $E_{1 / 2}$ provide an additional support of important role of electron transfer in the mechanism of antioxidant activity of carotenoids. These experiments show that GA itself displays a substantial antioxidant activity with $\mathrm{k} / \mathrm{k}_{\mathrm{ST}}=12$ (Table 1). Note that the rate constant of the reaction between GA and peroxyl radicals is independent on GA concentration. On the other hand, a strong dependence of the rate constants on the concentration of GA was detected for carotenoid complexes. This fact is in accordance with earlier established changes of the structure of glycyrrhizin self-associates at the concentration range above $1 \mathrm{mM}$ [46], and preclinical studies with the rat models [55-56].

\section{COMPLEX OF GLYCYRRHIZIN WITH NIFEDIPINE}

One of the most challenging tasks of modern medicinal chemistry involves the attempts to increase drug efficiency, at the same time, lowering its toxicity. A promising way to create novel and more powerful forms is the preparation of molecular complexes of the existing drugs with known complexing agents. Such agents are able to facilitate drug delivery, to protect parent substances from metabolic decay, and to contribute to their prolonged action. For example, the complex of GA with nifedipine (NF, 1,4-dihydro-2,6dimethyl-4-(2'-nitrophenyl)-3,5-pyridinedicarboxylic acid dimethylester, see Scheme 1) shows significant increase in therapeutic activity on the models of adrenaline-induced hypertension and $\mathrm{CaCl}_{2}$ induced arrhythmia [56]. In the preclinical study of nifedipine activity using the model of $\mathrm{CaCl}_{2}$ induced arrhythmia in rats, it has been shown, that minimum therapeutic dose of NF-GA complex is 29 times lower as compared to free NF. To understand the reasons underlying the above-mentioned interplay between toxicity and therapeutic activity of nifedipine, two different hypotheses were discussed [34]. First hypothesis can be drawn from the recently reported strong influence of GA on the HSA (human serum albumin) binding of NF [59]. Since GA protects NF from binding to HSA, this can result in increase of free NF concentration. The second hypothesis is the influence of complexation on metabolism rate of the drug at the binding site of corresponding receptor [34]. It has been described in [60] the potential model of drug-receptor interaction where chemical transformations of the drug were a key step in the dissociation of the drug-receptor complex. This model was developed by the example of the calcium channel blocker nifedipine [60].

NF is known to bind with the "closed state" of active site of the L-type calcium receptors comprised of six spatially separated amino acid residues. Since detailed computational

Table 1. Relative Rate Constants of OOH Radicals Scavenging by Glycyrrhizic Acid, Carotenoids, and their Complexes (k/k $\left.\mathrm{k}_{\mathrm{ST}}\right)$ in DMSO. $E_{1 / 2}$ are the Redox Potentials of Carotenoids (in V vs. SCE). $k_{S T}$ is the Scavenging Rate Constant by Spin Trap PBN Applied for this Experiment

\begin{tabular}{|c|c|c|c|c|}
\hline $\begin{array}{c}{[\mathrm{GA}]} \\
\mathbf{m M}\end{array}$ & GA & $\begin{array}{c}\text { Zeaxanthin } \\
\left(\mathbf{E}_{1 / 2}=0.56 \mathrm{~V}\right)\end{array}$ & $\begin{array}{l}\text { Canthaxanthin } \\
\left(E_{1 / 2}=0.68 \mathrm{~V}\right)\end{array}$ & $\begin{array}{c}\text { 7'-apo-7',7'-dicyano- } \beta \text {-carotene } \\
\left(\mathbf{E}_{1 / 2}=0.72 \mathrm{~V}\right)\end{array}$ \\
\hline 0 & & 4 & 2 & 7 \\
\hline 0.5 & 12 & 4 & 59 & 133 \\
\hline 1 & 12 & 4 & 46 & 116 \\
\hline
\end{tabular}


analysis [62] demonstrated noticeable charge transfer between tyrosine residual and NF in the receptor's binding site, it has been proposed the single electron transfer as the potentially most probable step during the interaction of $\mathrm{NF}$ with the $\mathrm{Ca}^{2+}$ receptor binding site [60]. The investigation of the model reaction - photoinduced interaction of NF with Tyr in the presence and absence of GA in solution by using CIDNP method has shown that the inclusion of NF into the complex with GA significantly decrease the rate of single electron transfer [34] (Detailed description is done in chapter 4.2). Decrease in the rate of the drug-receptor interaction, might prolong the effective retention time of the drug linked to the membrane receptor.

\subsection{Solubility of Nifedipine-Glycyrrhizin Complex}

Physicochemical study of nifedipine-glycyrrhizin complex was performed by examining the solubility diagram of NF and by measuring the spin-spin relaxation time $\mathrm{T}_{2}$ of NF protons in the absence and in the presence of GA [34]. The longitudinal (or spin-lattice) relaxation rate $\left(1 / \mathrm{T}_{1}\right)$ and the transverse (or spin-spin) relaxation rate $\left(1 / \mathrm{T}_{2}\right)$ are NMR parameters that may additionally be used to measure binding. Three characteristic regions are evident from the solubility diagram: (1) an increase of the NF concentration, (2) a plateau, and (3) the subsequent concentration growth. The observed solubility pattern allows us to suggest that the variation of GA concentrations results in the formation of differently organized complexes. Remarkably, the concentration points in the solubility diagram where step changes in the solubility of the NF are observed (Fig. 3) correspond to the earlier detected jump-in points in the dynamic viscosity of GA solutions [23] and the fluorescence intensity of carotenoids in GA complex [46]. Since recent NMR study of GA solutions has demonstrated the formation of GA micelles at concentrations higher $1 \mathrm{mM}$ [28-29], the presented solubility diagram allows concluding that micelle formation results in further increase of NF solubility.

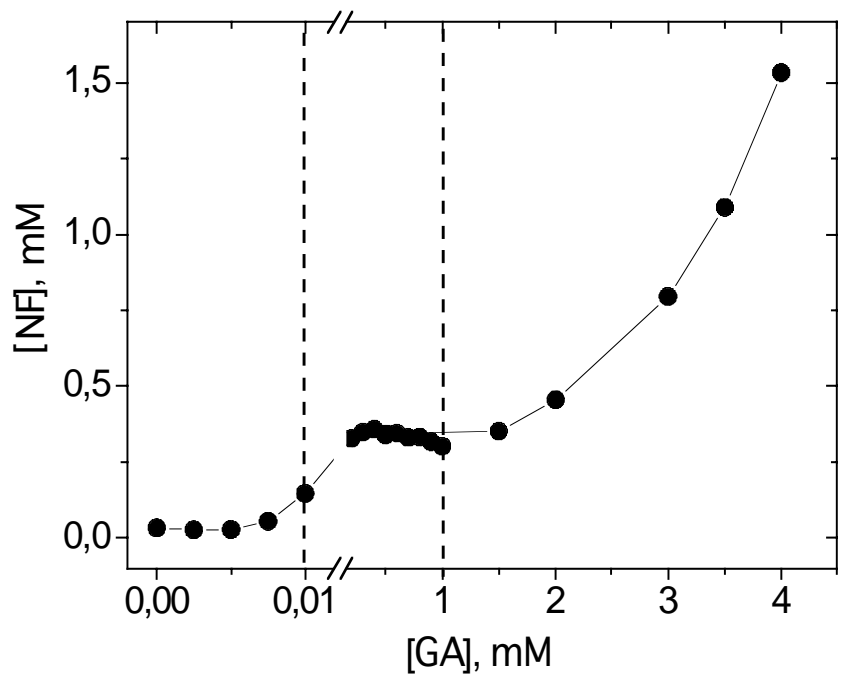

Fig. (3). The solubility diagram of nifedipine in the presence of GA in 5\% methanol aqueous solution. Adopted from [34].

The stoichiometry of the complex measured by Job's Plot at middle concentration range $(0.5 \mathrm{mM})$ provided the same complex structure as it was defined for carotenoid complexes: NF-GA 2 [34] (see Fig. 3). The value of stability constant determined in [34], $\mathrm{K}=1.2 \times 10^{5} \mathrm{M}^{-1}$, points to

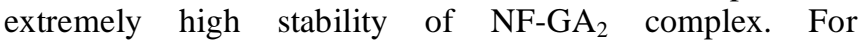
comparison, the stability constants of cyclodextrin complexes, widely used in pharmacology, are on the average $10^{3} \mathrm{M}^{-1}$, two orders of magnitude lower than the values observed for GA complexes [2].

Relaxation measurements were performed for $2,6-\mathrm{CH}_{3}$ $\mathrm{NF}$ protons in pure and $30 \%$ methanol solutions at GA concentration equal to $0.7 \mathrm{mM}$, below CMC. The values of $\mathrm{T}_{2}$ are equal $1.4 \pm 0.04 \mathrm{sec}$ for free $\mathrm{NF}$ in the absence of GA, $0.8 \pm 0.2 \mathrm{sec}$ for NF-GA 2 complex, and $0.06 \pm 0.02 \mathrm{sec}$ for $\mathrm{NF}$ in glycyrrhizin micelle [34]. We propose that high sensitivity of $\mathrm{T}_{2}$ values to drug conjugates formation makes this techniques an excellent instrument also for binding study of the drug molecules with biological supramolecules.

\subsection{Interaction of Nifedipine-Glycyrrhizin Complex with Amino Acid Residues}

Chemically Induced Dynamic Nuclear Polarization (CIDNP) technique was used to establish the detailed molecular mechanism of the interaction of NF with amino acid residues [60]. The model reaction in solution was initiated by UV irradiation. This approach is based on the assumption that reactivity of active intermediates would not depend on their generation pathway. It was proposed that the activation barrier in the drug-receptor complex is decreased through the charge-transfer process. It will result in the same effect as photoinitialization. It has been established that the mechanism includes three basic steps: (i) the formation of the neutral radical 1 resulting from sequential electron transfer from tyrosine to NF, followed by proton transfer; (ii) transformation of $\mathbf{1}$ into dihydropyridine neutral radical $\mathbf{2}$ after the elimination of water molecule; and (iii) electron or hydrogen atom transfer regenerating the initial tyrosine molecule and leading to the main reaction product. It is necessary to note that nifedipine metabolism in liver, under the action of cytochrome $\mathrm{P} 450$, leads to nitropyridine, rather than to nitrosopyridine.

The proposed mechanism of the model reaction of nifedipine with tyrosine allows to formulate the general concept of the processes which occur in the active site of receptor during the formation of the complex with nifedipine. As it has been mentioned above, the stability of this complex is defined by several determinants. First, it is the binding of the electron-rich dihydropyridine cycle with three amino acids of the active site; second, it includes the donor-acceptor interaction between three other amino acid residue and nitrophenyl substituent which is believed to be accompanied by charge-transfer interaction [62, 63]. It was established that the nifedipine radical anion formed by electron transfer from tyrosine is unstable particle and rapidly transforms (in microseconds time scale) into final product - nitrosopyridine. Nitrosopyridine could not be bound to the receptor active site, and the complex dissociates, thus, unlocking the receptor. As it was mentioned above, complexation with GA inhibits electron transfer step [34]. This results in the drop of the product yield and, in turn, might prolong the therapeutic action of the drug. It should be mentioned that high photosensitivity of NF [64] and formation of free radical intermediates [60] might 

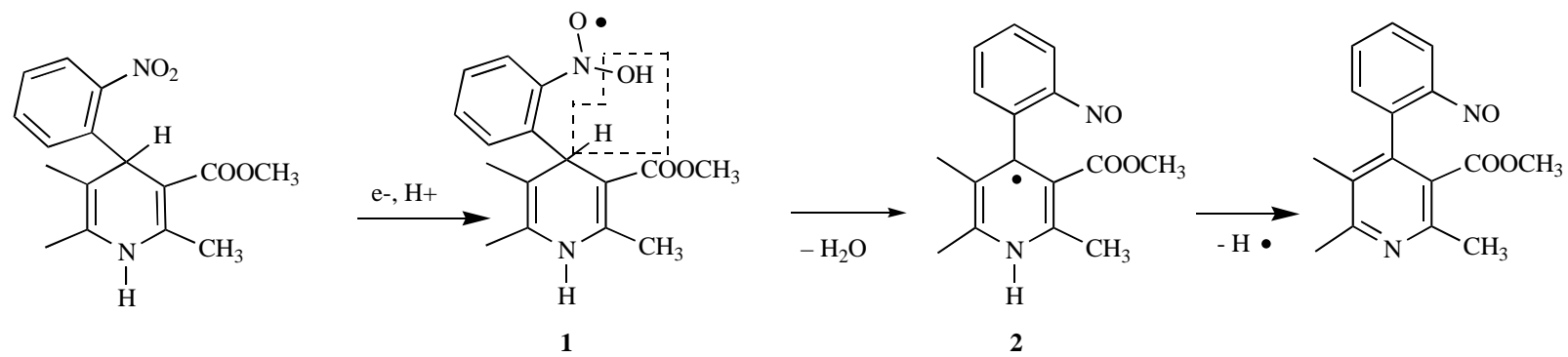

Scheme 1. Proposed mechanism of the interaction of nifedipine with amino acid tyrosine. Adopted from [60].

be the reasons of high phototoxicity of nifedipine. The using of GA complexes of NF instead of free NF will results in significant decrease of toxicity of this drug, including phototoxicity (from both, reducing therapeutic dose and reducing reactivity).

\section{LAPPACONITINE COMPLEX WITH GLYCYR- RHIZIC ACID}

Lappaconitine (LA), diterpene alkaloid from Aconitum septentrionale Koelle (Ranunculaceae), (Fig. 4) was the second example where the participation of electron transfer stage in the process of ligand-receptor interaction was suggested and confirmed using physicochemical approach [61]. It is known that lappaconitine reveals bradycardic and hypotensive activity [64-65]. It was demonstrated using CIDNP technique, that in the presence of amino acids tyrosine and tryptophan the reaction occurs via formation of intermediate lappaconitine radical anion with following proton transfer and ether $\mathrm{C}(\mathrm{O}) \mathrm{O}-\mathrm{R}$ bond cleavage. Similar to nifedipine radical anion, radical anion of lappaconitine is unstable and rapidly transforms into reaction products which are not the $\mathrm{Na}$ ion channel blockers. Note that the knowledge about short lived paramagnetic intermediates of lappaconitine is very important because of its high toxicity, including phototoxicity [66-67]. There is high probability that the side effects of LA are connected with formation of its paramagnetic forms. Since the influence of glycyrrhizic acid on therapeutic activity of lappaconitine was established [14], the physicochemical study of LA-GA complex was carried out to understand the molecular mechanism of this effect [29, 68]. In addition, the influence of GA on the efficiency and separate stages of lappaconitine phototransformation has been investigated in these studies.

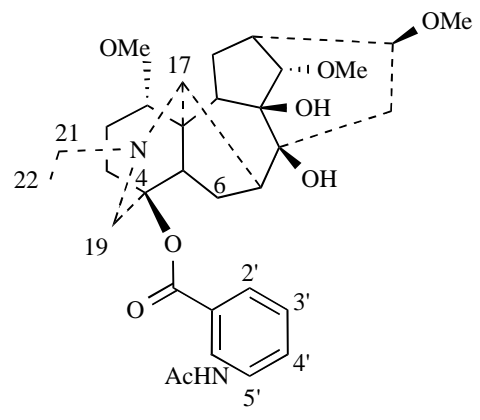

Lappaconitine (LA)

Fig. (4). The structures of lappaconitine.

Complexation of LA with glycyrrhizic acid was investigated using optical absorption, NMR and CIDNP techniques $[29,68]$. The stability constants of LA-GA complexes were determined equal to $2 \times 10^{5} \mathrm{M}^{-1}$ in wateralcohol solution, $1 \times 10^{4} \mathrm{M}^{-1}$ in methanol solution, and $1 \times$ $10^{3} \mathrm{M}^{-1}$ for water-alcohol solution of lappaconitine hydrobromide [68].

\subsection{Paramagnetic Intermediates of Lappaconitine and the Influence of GA on its Reactivity}

Now we describe the application of the approach based on the combination of NMR and CIDNP methods to study the radical transformation of LA and the influence of GA on the separate stages of this reaction [29]. It was established that photochemistry of esters of anthranilic acid is strongly dependent on H-bond formation. Intramolecular hydrogen bonding inhibits any photochemical transformations. On the contrary, esters which form intermolecular H-bond with proton donor solvents react via triplet state under UV irradiation. According to CIDNP data the photolysis of Lap in polar water-methanol mixture in the absence of GA shows two alternative pathways (Scheme 2). The pathway "A" presents the process of the radical deacylation without cleavage of another bond in the molecule. The pathways " $\mathrm{B}$ " and " $C$ " correspond to the photocleavage with breaking of ester bond in monomolecular and bimolecular reactions.

The substantial broadening and shift of lines of aromatic protons in NMR spectra of LA has been observed in the presence of GA. These broadening points to an increase in the relaxation rate of LA protons in the presence of GA. The reason of this effect might be the restriction of mobility of LA molecule due to penetration inside GA micelles. Besides that the solubilization in the micelle might displace water from nearest environment of LA. It results in replacement of the intermolecular hydrogen bonds of carbonyl group with water to intramolecular one with protons of amine group. Fig. (5) shows the influence of GA on polarization intensity in pathways A and B. Since CIDNP intensity is proportional to the radical pair concentration, the observed effect indicates that the complexation with GA almost fully blocks both reaction pathways.

Thus, presented results demonstrate strong influence of organized media on lappaconitine radical phototransformation. Note that these results also might be important for medical practice, because of LA demonstrates phototoxicity.

\subsection{The Influence of GA on the Reaction of Lappaconitine with Amino Acids}

The influence of GA on the reaction of photoinduced electron transfer between lappaconitine and amino acids tyrosine and tryptophan, which present in the peptide loop of 


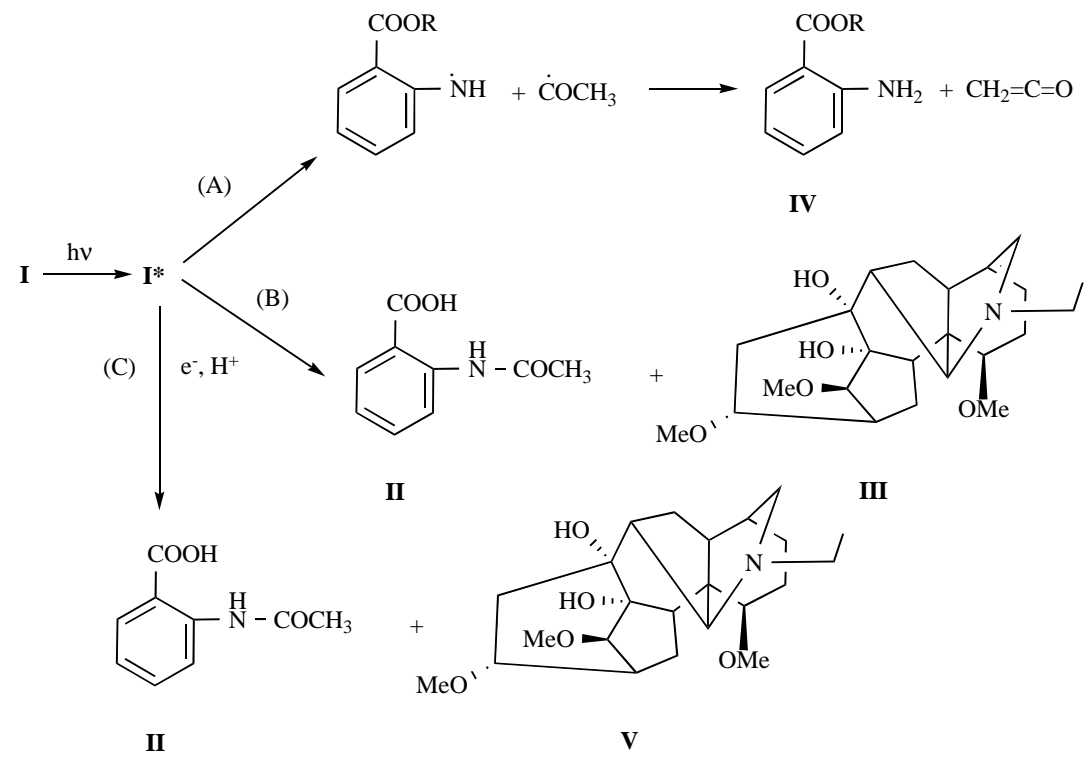

Scheme 2. Proposed mechanisms of lappaconitine (I) phototransformation: Adopted from [29].

site 2 of the sodium channel, was also investigated by NMR and CIDNP techniques [68]. The primary conclusion to be drawn from these experiments is that the presence of GA decreases significantly the rate of electron transfer. The polarization intensity and the yield of the reaction product (II) decrease by factor of 4 in the presence of GA. Note, that this experiments were carried out under condition when no GA micelles was formed. This result testifies in favor of the suggested mechanism of the influence of GA on the therapeutic activity of lappaconitine and the role of chemical reactions in the dissociation of ligand-receptor complexes.

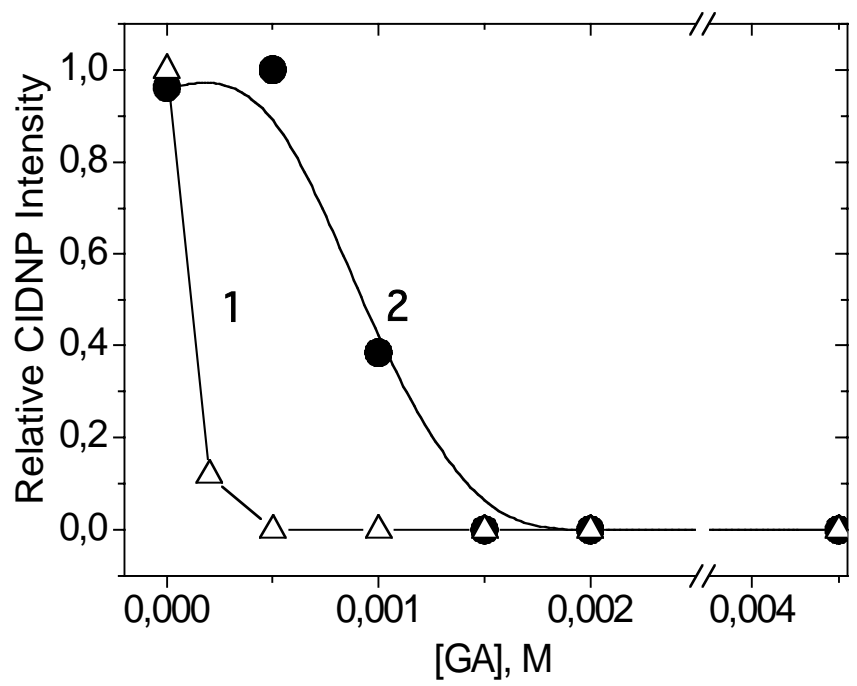

Fig. (5). Dependence of the relative CIDNP intensity of 5'-H of IV (2) and 19-H of III (1) protons detected during the photolysis of LA in $\mathrm{CD}_{3} \mathrm{OD}$ aqueous solution. Adopted from [29].

\section{GLYCYRRHIZIN COMPLEX WITH HYPOCHO- LESTEROLEMIC DRUG SIMVASTATIN}

Simvastatin belongs to a class of statins, the well known inhibitors of 3-hydroxi-3-methylglutaril-CoA reductase (3HMG-CoA reductase), widely used in anti-atherosclerosis therapy [69]. However, most statins in effective therapeutic dose gives rise to unwanted side effects - liver toxicity, myalgia, myopathy and in rare cases - rhabdomyolysis [70]. Therefore, the search for and creation of new formulations with a lower dose, more secure, long-acting and effective is an actual task. One of the modern approaches to achieve this goal is preparation of supramolecular complexes of existing statins. The positive result was obtained by using of glycyrrhizic acid as a complexing agent for simvastatin [7173].

To prove the complex formation between simvastatin and glycyrrhizin, an optical absorption and NMR techniques were applied [71]. The noticeable changes in simvastatin absorption spectrum were detected in the presence of GA. From the concentration dependence of optical density of simvastatin solution (Benesi-Hildebrand plot [52], D/ $\Delta \mathrm{D}-1$ $\left.=1 /[\mathrm{GA}]^{\mathrm{n}} \times 1 / \mathrm{K}\right)$ the stoichiometry and stability constant of the complex were calculated. The result of this study shows that this complex consists of one simvastatin molecule and four glycyrrhizin molecules. The stability constant of this complex was determined as $\mathrm{K}=3 \times 10^{14} \mathrm{M}^{-4}$.

This complex was entitled "simvaglysin", and its properties were investigated by means of "in vitro" and "in vivo" methods [71]. The investigation of the inhibition of HMG-CoA reductase from microsomal fraction of rat liver by simvaglysin has shown that this complex works via uncompetitive mechanism, while free simvastatin demonstrates competitive inhibition. Also simvaglysin manifested cytochrom P450 depending type of inhibition contrary to free simvastatin which shows esterase depending inhibition. The change of the mechanism of statin activity is the most important feature of this GA complex. Effective even at the doses containing $1 / 5$ of statin's normal dose, "simvaglyzin" is a more effective and safer drug as compared with free simvastatin.

\section{CONCLUSION}

The formation of various GA self associates and their conjugation with drugs was confirmed by different physical 
methods. The complexes of GA dimers with drug molecules were observed at low GA concentrations $\left(10^{-3}-10^{-5} \mathrm{M}\right)$. At higher concentration $\left(\geq 10^{-3} \mathrm{M}\right) \mathrm{GA}$ forms large micelle-like aggregates. This review demonstrated that all drugs under study (nifedipine, lappaconitine, carotenoids and simvastatin) reveal new qualities, when they are incorporated in the complexes and micelles of GA. The results presented in this review make us to consider glycyrrhizic acid as a prospective compound for using as novel drug delivery system, drug stabilizer as well as enhancer of drug activity. In addition, the unique possibility of CIDNP method to elucidate the structure of the radical drug intermediates has been demonstrated.

\section{ACKNOWLEDGEMENT}

The financial support of Russian Foundation for Basic Research, grant 08-03-00372, priority program of Russian academy of science "Physicochemical study of supramolecular complexes of plant metabolites with hypocholesterinemic agents", and the U.S. Department of Energy, Grant DE-FG02-86ER13465 are gratefully acknowledged.

\section{REFERENCES}

[1] Loftsson, T.; Brewester. M. Pharmaceutical applications of cyclodextrins. 1. Drug solubilization and stabilization. J. Pharm. Sci., 1996, 85, 1017-1025. a. Szejtli, J. Introduction and General Overview of Cyclodextrin Chemistry. Chem. Rev., 1998, 98, 17431754.

[2] Singh, M.; Sharma, R.; Banerjee, U. C. Biotechnological applications of cyclodextrins. Biotechnol. Adv., 2002, 20, 341-359.

[3] Uekama, K.; Hirayama, F.; Irie, T. Cyclodextrin drug carrier systems. Chem. Rev., 1998, 98, 2045-2076.

[4] Stella, V. J.; Rajewski, R. A. Cyclodextrins: Their future in drug formulation and delivery. Pharm. Res., 1997, 14, 556-567.

[5] Szejtli J. Cylodextrin in drug formulations: Part I. Pharm. Technol. Int., 1991, 3, 15-23.

[6] Tolstikov, G.A.; Baltina, L.A.; Grankina, V.P.; Kondratenko, R.M.; Tolstikova, T.G. Licorice: biodiversity, chemistry and application in medicine, Academic Publishing House "GEO": Novosibirsk, 2007.

[7] Tolstikov, G.A.; Baltina, L.A.; Schults, E.E.; Pokrovsky, A.G. Glycyrrhizic acid. Bioorg. Chem., 1997, 23, 691-709.

[8] Dushkin, A.V.; Karnatovskaya, L.M.; Chabueva, E.N.; Boldyrev, V.V.; Sorokina, I.V.; Tolstikova, T.G.; Dolgikh, M.P.; Shults, E.E. Production and analysis of ulcerogenic action of quick-dissolving solid dispersed systems based on acetylsalicylic acid and biologically active licorice derivatives. Pharm. Chem. J., 2001, 11, 18-21.

[9] Bondarev, A.I.; Bashkatov, S.A.; Davydova, V.A.; Zarudiy, F.S.; Lazareva, D.N.; Tolstikova, T.G.; Baltina, L.A.; Tolstikov, G.A.; Murinov, Y.I.; Rusakov, I.A. Antiinflammatory and analgesic action of complexes of antiflogistics with glycyrrhizic acid. Pharmacol. Toxicol., 1991, 5, 47-50.

[10] Baltina, L.A.; Davydova, V.A.; Tolstikova, T.G.; Sharipova, F.V.; Murinov, Yu.I.; Zarudiy, F.S.; Lazareva, D.N.; Tolstikov, G.A.; Bondarev, A.I. Complex compound acetylsalicylic acid with glycyrrhizin, which manifest anti-inflammatory, antipyretic and antiulcerous activity. USSR Patent 1566700, May 23, 1990.

[11] Baltina, L.A.; Sharipova, F.V.; Davydova, V.A.; Tolstikova, T.G.; Zarudiy, F.S.; Lazareva, D.N.; Tolstikov, G.A.; Bondarev, A.I. Complex compound of 1-phenyl-2 ,3-dimethyl-4-methyl-5aminopirazolon sodium metasulfat with glycyrrhizin, exhibiting anti-inflammatory and analgesic activity. USSR Patent 1566696, May 7, 1991.

[12] Baltina, L.A.; Davydova, V.A.; Tolstikova, T.G.; Murinov, Yu.I.; Lazareva, D.N.; Tolstikov, G.A.; Bondarev, A.I. Complex compound of the sodium salt of 2 - [(2,6-dichloro-phenyl) amino] phenyl acetic acid with glycyrrhizic acid, exhibiting antiinflammatory activity. USSR Patent 1566699, May 7, 1991.

[13] Bryzgalov, A.O.; Tolstikova, T.G; Sorokina, I.V.; Zhukova, N.A.; Volkova, E.B.; Dolgikh, M.P. Antiarrhythmic activity of alaglizin. J. Expert Clin. Pharm., 2005, 68, 24-28.
[14] Yunusov, M.S.; Tolstikov, G.A.; Murinov, Yu.I.; Tsyrlina, E.M.; Tolstikova, T.G.; Sorokina, I.V.; Voevoda, T.V.; Yunusova, S.G.; Dokichev, V.A.; Kaverina, N.V.; Turilova, A.I. Medication to treat various forms on the fracture of heart rhythm. RF Patent 2180583, March 20, 2002.

[15] Pogosova, G.V. Nifedipine in treatment of cardiovascular system deseases: new about well known. Clin. Pharm. Ther., 2006, 3, 2-6.

[16] Tolstikova, T.G.; Khvostov, M.V.; Bryzgalov, A.O. The Complexes of Drugs with Carbohydrate-Containing Plant Metabolites as Pharmacologically Promising Agents. Mini-Rev. Med. Chem., 2009, 9, 1317-1328.

[17] James, K.C.; Stanford, J.B. The solubilising properties of liqurica. J. Pharm. Pharmacol., 1962, 5, 445-450.

[18] Gilbert, R.J.; James, K.C. Preparation and surface-active properties of glycyrrhizic acid and its salts. J. Pharm. Pharmacol., 1964, 16, 394-399.

[19] Azaz, E.; Segal, R. Glycyrrizin as gelling agent. Pharm. Acta Helv. 1980, 55, 183-186.

[20] Kondo, M.; Minamino, H.; Okiyama, G. Physicochemical properties and applications of $\alpha$ - and $\beta$-glycyrrhizins, natural surface active agents in licorice root extract. J. Soc. Cosmet. Chem., 1986, 37, 177-189.

[21] Maskan, M. Rheological behavior of liqorice (Glycyrrhiza glabra) extract. J. Food Process Eng., 1999, 39, 389-393.

[22] Romanenko, T.V.; Murinov, Yu.I. Some features of diluted glycyrrhizic acid solutions flux. J. Phys. Chem., 2001, 75, 16011604.

[23] Soltesz, G.; Uri, G. Solubilisierende Wirkung des Monoammonium glycyrrhizinats auf Antibiotika und einige andere Stoffe. Naturwissenschaften, 1963, 50, 691.

[24] Krasova, T.G.; Bashura, G.S.; Murav'ev, I.A. Hydrocortisone and prednisolone solubilization in aqueous solutions of glyciram. Pharmacy, 1978, 27, 32-35.

[25] Sasaki, Y.; Mizutani, K.; Kasai, R.; Tanaka, O. Solubilizing properties of glycyrrhizin and its derivatives: solubilization of saikosaponin-a, the saponin of bupleuri radix. Chem. Pharm. Bull, 1988, 36, 3491.

[26] Tolstikov, G.A.; Murinov, Y.I.; Baltina, L.A. Complexes of $\beta$ glycyrrhizic acid with drugs as a novel transport forms. Pharm. Chem. J., 1990, 24, 26-27.

[27] Kornievskaya V.S., A.I. Kruppa, T.V. Leshina, NMR and photoCIDNP investigations of the glycyrrhizinic acid micelles influence on solubilized molecules. J. Incl. Phen. Macrocycl. Chem., 2007, 60, 123-130.

[28] Kornievskaya V. S., Kruppa A. I., Polyakov N. E., and Leshina T. V. Effect of Glycyrrhizic Acid on Lappaconitine Phototransformation. J. Phys. Chem. B, 2007, 111, 11447-11452.

[29] H.-J. Schneider, F. Hacket, V. Rudiger, NMR studies of cyclodextrins and cyclodextrin inclusion complexes. Chem. Rev., 1998, 98, 1755-1785.

[30] Emsley, J.W., Freeney, J., Sutcliffe, L.H. High Resolution Nuclear Magnetic Resonance Spectroscopy, Pergamon Press, Oxford, 1965, Vol. 1 .

[31] Mao, S.Z., Zhang, X.D., et al.: Nuclear magnetic resonance relaxation studies of polyacrylamide solution. Colloid. Polym. Sci., 2000, 278, 264-269.

[32] Popova, M.V., Tchernyshev, Y.S., Michel, D.: NMR Investigation of the Short-chainIonic Surfactant-Water Systems. Langmuir, 2004, 20, 632-636.

[33] Polyakov, N.E.; Khan, V.K.; Taraban, M.B.; Leshina, T.V. Complex of Calcium Receptor Blocker Nifedipine with Glycyrrhizic Acid. J. Phys. Chem. B, 2008, 112, 4435-4440.

[34] Kispert, L.D. and Polyakov, N.E. Carotenoid radicals. Cryptochemistry of Natural Colorants. Chem. Lett., 2010, 39, 148155.

[35] El-Agamey, A.; Lowe, G. M.; McGarvey, D. J.; Mortensen, A.; Phillip, D. M.; Truscott, T. G.; Young, A.J. Carotenoid radical chemistry and antioxidant/pro-oxidant properties. Arch. Biochim. Biophys., 2004, 430, 37-48.

[36] Palozza, P.; Calviello, G.; Serini, S.; Maggiano, N.; Lanza, P.; Ranelletti, F. O.; Bartoli, G. M. Beta-carotene at high concentrations induces apoptosis by enhancing oxy-radical production in human adenocarcinoma cells. Free Radic. Biol. Med., 2001, 30, 1000-1007.

[37] Cross, C. E.; Halliwell, B.; Borish, E.T. Oxygen radicals and human disease. Ann. Int. Med., 1987, 107, 526-545.

[38] Halliwell, B. Antioxidants and human disease: A general introduction. Nutr. Rev. 1997, 55, 44-52.

[39] Polyakov, N.E.; Kispert, L.D. In: Beta Carotene: Dietary Sources, Cancer and Cognition. Eds. by L. Haugen and T. Bjornson, Nova Science Publishers, 2009, pp. 191-230. 
[40] Mele, A.; Mendichi, R.; Selva, A.; Molnar, P.; Toth, G. Noncovalent associations of cyclomaltooligosaccharides (cyclodextrins) with carotenoids in water. A study on the alpha- and betacyclodextrin/psi,psi-carotene (lycopene) systems by light scattering, ionspray ionization and tandem mass spectrometry. Carbohydr. Res., 2002, 337, 1129-1136.

[41] Szente, L.; Mikuni, K.; Hashimoto, H.; Szejtli, J. Stabilization and solubilization of lipophilic natural colorants with cyclodextrins. $J$. Inclusion Phenom. Mol. Recognit. Chem. 1998, 32, 81-89.

[42] Lancrajan, I.; Diehl, H. A.; Socaciu, C.; Engelke, M.; Zorn-Kruppa, M. Carotenoid incorporation into natural membranes from artificial carriers: liposomes and beta-cyclodextrins. Chem. Phys. Lipids, 2001, 112, 1-10.

[43] Polyakov, N.E.; Leshina, T.V.; Konovalova, T.A.; Kispert, L.D. Inclusion Complexes of Carotenoids with Cyclodextrins. ${ }^{1} \mathrm{H}$ NMR, EPR and Optical Studies. Free Radic. Biol. Med., 2004, 36, 872880.

[44] Lockwood, S.F.; O'Malley, S.; Mosher G.L. Improved aqueous solubility of crystalline astaxanthin (3,3'-dihydroxy- $\beta, \beta$-carotene$4,4^{\prime}$-dione) by Captisol ${ }^{\circledR}$ (sulfobutyl ether $\beta$-cyclodextrin). $J$. Pharm. Sci., 2003, 92, 922-926.

[45] Polyakov, N.E.; Leshina, T.V.; Salakhutdinov, N.F.; Kispert, L.D. Host-Guest Complexes of Carotenoids with $\beta$-Glycyrrhizic Acid. $J$. Phys. Chem. B. 2006, 110, 6991-6998.

[46] Polyakov, N.E.; Leshina, T.V.; Salakhutdinov, N.F.; Konovalova, T.A. and Kispert, L.D. Antioxidant and Redox Properties of Supramolecular Complexes of Carotenoids with $\beta$-Glycyrrhizic Acid. Free Radic. Biol. Med., 2006, 40, 1804-1809.

[47] Polyakov, N.E.; Leshina, T.V.; Meteleva, E.S.; Dushkin, A.V.; Konovalova, T.A. and Kispert, L.D. Water Soluble Complexes of Carotenoids with Arabinogalactan. J. Phys. Chem. B, 2009, 113, 275-282.

[48] Polyakov, N. E.; Leshina, T. V.; Meteleva, E. S.; Dushkin, A. V.; Konovalova, T. A.; and Kispert, L. D. Enhancement of Photocatalytic Activity of $\mathrm{TiO}_{2}$ Nanoparticles by Water Soluble Complexes of Carotenoids. J. Phys. Chem. B, 2010, 114, 1420014204 .

[49] Gusakov, V. N.; Maistrenko, V. N.; Safiullin, P. P. Thermodynamics of Formation of Molecular Complexes of Aromatic Nitro Derivatives with Lincomycin and $\beta$-Glycyrrhizic Acid. Russ. J. Gen. Chem. 2001, 71, 1307-1310.

[50] Maistrenko, V.N.; Gusakov, V.N.; Rusakov, I.A.; Murinov, Yu.I.; Tolstikov, G.A. Complexation of glycyrrhizinic acid with 5-nitro8-oxiquinoline and trinitroglycerine. Dokl. Akad. Nauk, 1994, 335, 329-331 (in Russian).

[51] Lopez, E. A.; Bosque-Sendra, J. M.; Rodriguez, L. C.; Campana, A. M. G.; Aaron, J. J. Applying non-parametric statistical methods to the classical measurements of inclusion complex binding constants. Anal. Bioanal. Chem., 2003, 375, 414-423.

[52] Rekharsky, M. V.; Inoue, Y. Complexation Thermodynamics of Cyclodextrins. Chem. Rev., 1998, 98, 1875-1917.

[53] Maafi, M.; Mahedero, M.C.; Aaron, J.J. Fluorimetric properties of a 2-hydroxypropyl-beta-cyclodextrin: 9-methyl-benzo[a] phenothiazine inclusion complex in aqueous media. Analytical usefulness. Talanta, 1997, 44, 2193-2199.

[54] Tolstikova, T.G.; Sorokina, I.V.; Bryzgalov, A.O.; Dolgikh, M.P.; Lifshits, G.I.; Khvostov, M.V. The usage of new approach of known drugs complexation with plant glycosides in prophylaxis and relief of acute arterial hypertension (experimental study). $J$. Rational Pharmacother. Cardiol., 2006, 1, 55-58.

[55] Tolstikova, T. G.; Khvostov, M. V.; Bryzgalov, A. O.; Dushkin, A. V.; Meteleva, E. S. Complex of Nifedipine with Glycyrrhizic Acid as a Novel Water-Soluble Antihypertensive and Antiarrhythmic Agent. Lett. Drug Des. Discov., 2009, 6, 155-158.

[56] Polyakov, N.E.; Kruppa, A.I.; Leshina, T.V.; Konovalova, T.A.; Kispert, L.D., The Carotenoids as Antioxidants. Spin-Trapping ESR and Optical Study. Free Radic. Biol. Med., 2001, 31, 43-52.
[57] Polyakov, N.E.; Leshina, T.V.; Konovalova, T.A.; and Kispert, L.D. Carotenoids as Scavengers of Free Radicals in a Fenton Reaction, Antioxidants or Pro-oxidants. Free Rad. Biol. Med. 2001, 31, 398-404

[58] Lee, K.-J.; Park, H.-J.; Shin, Y.-H.; Lee, C.-H. Effect of glycyrrhizic acid on protein binding of diltiazem, verapamil, and nifedipine. Arch. Pharm. Res., 2004, 27, 978-983.

[59] Polyakov, N.E.; Taraban, M.B.; Leshina, T.V. Photo-CIDNP Study of the Interaction of Tyrosine with Nifedipine. An Attempt to Model the Binding between Calcium Receptor and Calcium Antagonist Nifedipine. Photochem. Photobiol., 2004, 80, 565-571.

[60] Polyakov, N. E.; Khan, V. K.; Taraban, M. B.; Leshina, T. V.; Luzina, O. A.; Salakhutdinov N. F. and Tolstikov, G. A. Mechanisms of photoinduced electron transfer reactions of lappaconitine with aromatic amino acids. Time-resolved CIDNP study. Org. Biomol. Chem., 2005, 3, 881-885.

[61] Schleifer, K.-J. Stereoselective Characterization of the 1,4 Dihydropyridine Binding Site at L-Type Calcium Channels in the Resting State and the Opened/Inactivated State. J. Med. Chem., 1999, 42, 2204-2211.

[62] Schleifer, K.-J. Correlation of LUMO energies and free energies of binding for a series of nifedipine analogues. Pharmazie, 1999, 54, 804-807.

[63] Ono, M.; Satoh, T. Pharmacological studies on lappaconitine: antinociception and inhibition of the spinal action of substance $\mathrm{P}$ and somatostatin. Jpn. J. Pharmacol., 1991, 55, 523-530.

[64] Osadchii, S.A.; Pankrushina, N.A.; Shakirov, M.M.; Shults, E.E.; Tolstikov, G.A. Study of alkaloids from plants of Siberia and Altai 4. N-deethylation of diterpene alkaloids of the aconitane type. Russ. Chem. Bull., 2000, 3, 557-562.

[65] Polyakov, N.E.; Leshina, T.V.; Tkachev, A.V.; Nikitina, I.A Pankrushina, N.A. Phototransformation products of alkaloid lappaconitine: multinuclear NMR study. J. Photochem. Photobiol., A: Chem., 2008, 197, 290-294.

[66] Polyakov, N.E.; Simaeva, O.A.; Taraban, M.B.; Leshina, T.V.; Konovalova, T.A.; Kispert, L.D.; Nikitina, I.A.; Pankrushina, N.A.; Tkachev, A.V. CIDNP and EPR Study of Phototransformation of Lappaconitine Derivatives in Solution. J. Phys. Chem. B, 2010, $114,4646-4651$

[67] Polyakov, N. E.; Khan, V. K.; Taraban, M. B.; Leshina, T. V.; Salakhutdinov, N. F.; Tolstikov, G. A., Complexation of lappaconitine with glycyrrhizinic acid. Structure, stability and reactivity studies. J. Phys. Chem. B, 2005, 109, 24526-24530.

[68] Expert Panel on Detection, Evaluation, and Treatment of High Blood Cholesterol in Adults: Executive Summary of the Third Report of the National Cholesterol Education, and Treatment of High Blood Cholesterol in Adults (Adult Treatment Panel III). JAMA, 2001, 285, 2486-2497.

[69] Puddu, P.; Puddu, G.M.; Muscari, A. Acta Cardiol., 2001, 56, 225 231.

[70] Taraban, M.B.; Leshina, T.V.; Stakhneeva, E.M.; Lyakhovich, V.V.; Nikitin, Yu.P.; Tolstikov, G.A. Hypocholesteremic properties of complex compound of simvastatin with glycerrhizic acid (simvaglizin) in experimental models. Biomed. Chem., 2008, 54, 301-313.

[71] Tolstikov, G.A.; Nikitin, Yu.P.; Lyakhovich, V.V.; Salakhutdinov, N.F.; Ragino, Yu.I.; Vavilin, V.A. Drug with hypocholesterolemic effect Symvaglyzin. RF Patent 2308947, October 27, 2007.

[72] Ragino, Yu.I.; Vavilin, V.A.; Salakhutdinov, N.F.; Makarova, S.I.; Stakhneva, E.M.; Safronova, O.G.; Nikitin, Yu.P.; Tolstikov, G.A Examination of the hypocholesteremic effect and safety of simvaglizin on the model of hypercholesterolemia in rabbits. Bull.Exp. Biol. Med., 2008, 145, 285-287.

[73] Ragino, Yu.I.; Vavilin, V.A.; Salakhutdinov, N.F.; Makarova, S.I ; Stakhneva, E.M.; Safronova, O.G.; Nikitin, Yu.P.; Tolstikov, G.A. Study of cholesterol-lowering effect and safety of simvaglisin on rabbit model of hypercholesterolemia. Bull. Expr. Biol. Med., 2008, 145(3), 317-319.

(C) Polyakov and Leshina; Licensee Bentham Open.

This is an open access article licensed under the terms of the Creative Commons Attribution Non-Commercial License (http://creativecommons.org/licenses/by-nc/3.0/) which permits unrestricted, non-commercial use, distribution and reproduction in any medium, provided the work is properly cited. 